PROCEEDINGS OF THE

AMERICAN MATHEMATICAL SOCIETY

Volume 133, Number 2, Pages 545-556

S 0002-9939(04)07762-7

Article electronically published on September 16, 2004

\title{
POSITIVE SCALAR CURVATURE FOR MANIFOLDS WITH ELEMENTARY ABELIAN FUNDAMENTAL GROUP
}

\author{
BORIS BOTVINNIK AND JONATHAN ROSENBERG
}

(Communicated by Wolfgang Ziller)

\begin{abstract}
The statement often called the Gromov-Lawson-Rosenberg Conjecture asserts that a manifold with finite fundamental group should admit a metric of positive scalar curvature except when the $K O_{*}$-valued index of some Dirac operator with coefficients in a flat bundle is non-zero. We prove spin and oriented non-spin versions of this statement for manifolds (of dimension $\geq 5$ ) with elementary abelian fundamental groups $\pi$, except for "toral" classes, and thus our results are automatically applicable once the dimension of the manifold exceeds the rank of $\pi$. The proofs involve the detailed structure of $B P_{*}(B \pi)$, as computed by Johnson and Wilson.
\end{abstract}

\section{INTRODUCTION}

A fundamental and fascinating problem in global differential geometry is to determine necessary and sufficient conditions for a closed manifold to admit a Riemannian metric with scalar curvature function everywhere positive. For surveys on this question, see [17] and [15].

In this paper we consider this problem only for manifolds (of dimension at least five) with finite fundamental group. By a result of Kwasik and Schultz [9], the problem is then further reduced to the case where the fundamental group is a finite $p$-group. Somewhat different techniques are needed depending on whether or not the universal cover of the given manifold admits a spin structure. Here we will consider only the following two important cases: when the manifold admits a spin structure, and when it is oriented and its universal cover is not spin. (If $p$ is odd, these are the only two cases.)

When the universal cover is non-spin, there are no known obstructions to positive scalar curvature. In the spin case, the only known obstructions to positive scalar curvature come from the index theory of the Dirac operator ([14], [15]). In fact, in 12, it was conjectured (on the basis of extremely spotty evidence) that the Dirac operator index obstructions are the only obstructions to positive scalar curvature on manifolds of dimension $\geq 5$ with finite fundamental group. This conjecture has sometimes been called the Gromov-Lawson-Rosenberg Conjecture.

Received by the editors June 21, 2002.

2000 Mathematics Subject Classification. Primary 53C20; Secondary 53C21, 55S30, 55N22, $55 \mathrm{U} 25,57 \mathrm{R} 75$.

We thank Sergey Novikov for helping to make this collaboration possible.

This work was partially supported by NSF grant DMS-0103647.

(C)2004 American Mathematical Society Reverts to public domain 28 years from publication 
It is known that "stably" these are the only obstructions 14. This means that for such a manifold $M$ there exists an integer $k \geq 0$ such that the product $M \times\left(J^{8}\right)^{k}$ admits a positive scalar curvature metric if and only if the Dirac obstructions vanish. Here $J^{8}$ is a simply connected spin manifold with $\widehat{A}\left(J^{8}\right)=1$. Such a manifold $J^{8}$ does not admit positive scalar curvature and, geometrically, represents Bott periodicity in $K O$-theory. Moreover, $J^{8}$ can be chosen to be a Ricci-flat "Joyce manifold" 8] with holonomy Spin(7). The "stable" theorem by itself does not actually answer the question of whether any particular manifold with vanishing Dirac obstructions admits a metric of positive scalar curvature.

Botvinnik, Gilkey and Stolz [4] proved the Gromov-Lawson-Rosenberg Conjecture for spin manifolds with finite fundamental group with periodic cohomology. A similar theorem was proved by Schultz [16], and independently by Botvinnik and Gilkey [3], for spin manifolds of dimension $\geq 5$ with fundamental group $\mathbb{Z} / p \times \mathbb{Z} / p$, $p$ an odd prime. But very little was previously known about the existence of positive scalar curvature for manifolds with elementary abelian fundamental group $\pi=(\mathbb{Z} / p)^{r}$ of rank $r>2$. That is the subject of this paper. We build on some results of our earlier paper, [5]. Our main results are Theorems 2.3] and 2.4.

\section{MAIN RESULTS}

Let $\pi=(\mathbb{Z} / p)^{r}$ be an elementary abelian $p$-group, where we call $r$ the rank of $\pi$. We will be interested in the Gromov-Lawson-Rosenberg Conjecture for manifolds $M$ with fundamental group $\pi$. We will deal with the following cases:

(1) $p$ odd. The sub-case where $M$ is non-spin was already treated in [5], so we will concentrate here on the sub-case where $M$ is spin, though the same proof will work for the non-spin case, also.

(2) $p=2, M$ oriented, and the universal cover $\widetilde{M}$ is non-spin.

Recall that since any oriented (resp., spin) manifold is automatically oriented for ordinary homology (resp., connective or periodic real $K$-theory) that there are natural transformations $\Omega_{*}(\cdot) \rightarrow H_{*}(\cdot)$ and $\Omega_{*}^{\text {spin }}(\cdot) \rightarrow k o_{*}(\cdot)$. The composite

$$
\Omega_{*}^{\text {spin }}(X) \rightarrow k o_{*}(X) \rightarrow K O_{*}(X) \rightarrow K O_{*}(\text { point })
$$

corresponds to taking the generalized index of the Dirac operator and is traditionally denoted $\alpha$.

To state our main results, we have to single out certain bordism classes.

Definition 2.1. If $k \leq r$ and one fixes a homomorphism $\mathbb{Z}^{k} \rightarrow(\mathbb{Z} / p)^{k} \hookrightarrow(\mathbb{Z} / p)^{r}$, taking classifying spaces gives a map $T^{k}=B\left(\mathbb{Z}^{k}\right) \rightarrow B(\mathbb{Z} / p)^{r}$. The collection of all such maps is said to generate the subgroup of toral bordism classes in $\Omega_{k}(B \pi)$ or in $\Omega_{k}^{\text {spin }}(B \pi)$. We define a complement to the toral classes in the following way. Consider the natural Thom map $\Omega_{k}(B \pi) \rightarrow H_{k}\left(B \pi, \mathbb{F}_{p}\right)$ or $\Omega_{k}^{\text {spin }}(B \pi) \rightarrow$ $H_{k}\left(B \pi, \mathbb{F}_{p}\right)$, where $\mathbb{F}_{p}$ is the field of $p$ elements. When $p$ is odd, we have a natural cup product map $\bigwedge^{k} H^{1}\left(B \pi, \mathbb{F}_{p}\right) \hookrightarrow H^{k}\left(B \pi, \mathbb{F}_{p}\right)$, whose image (the group of toral cohomology classes) has dimension $\left(\begin{array}{l}r \\ k\end{array}\right)$. Even when $p=2$, even though in this case the classes in $H^{1}\left(B \pi, \mathbb{F}_{p}\right)$ do not have square 0 , we still have a similar subgroup of $H^{k}\left(B \pi, \mathbb{F}_{p}\right)$, spanned by products $\alpha_{i_{1}} \cdots \alpha_{i_{k}}, 1 \leq i_{1}<\cdots<i_{k} \leq r$, where $\alpha_{1}, \cdots, \alpha_{r}$ is a basis for $H^{1}\left(B \pi, \mathbb{F}_{p}\right)$. (The subgroup one gets in this case depends on the choice of basis.) A bordism class is called atoral if its image in $H_{k}\left(B \pi, \mathbb{F}_{p}\right)$ is 
annihilated by these toral cohomology classes. Toral and atoral classes in $H_{*}(B \pi)$ or $k o_{*}(B \pi)$ are defined to be the images of toral or atoral classes in bordism or spin bordism. Since the pairing between the toral homology and cohomology classes is non-degenerate, and since the non-zero toral bordism classes are all of order $p$ (since the bordism class of $S^{1} \rightarrow B \mathbb{Z} / p$ is of order $p$ ), the group of toral bordism classes is an elementary abelian $p$-group, and $\Omega_{k}(B \pi)$ or $\Omega_{k}^{\mathrm{spin}}(B \pi)$ splits as the direct sum of this group and the group of atoral classes.

Remark 2.2. (a) It is worth remarking, since we will need this observation later, that if $M^{k}$ is an oriented or spin $k$-manifold whose fundamental group can be generated by at most $k-1$ elements, then for any map $f: M \rightarrow B \pi$, the bordism class of $M \stackrel{f}{\rightarrow} B \pi$ is atoral.

(b) Another useful fact, which follows immediately from the definition, is that the group of toral classes (and when $p$ is odd, also the group of atoral classes) is preserved under injective group homomorphisms from one elementary abelian $p$-group to another.

Now we can state our main results:

Theorem 2.3. Let $p$ be an odd prime, let $\pi$ be an elementary abelian p-group, and let $n \geq 5$. Let $M$ be a spin manifold with $\alpha(M)=0$ in $K O_{n}$, and let $f: M \rightarrow B \pi$ be the classifying map for its universal covering. If the class $[M \stackrel{f}{\rightarrow} B \pi] \in \Omega_{n}^{\operatorname{spin}}(B \pi)$ is atoral, then $M$ has a metric with positive scalar curvature. In particular, if $n>$ $\operatorname{rank} \pi$, then every spin $n$-manifold with vanishing $\alpha$-invariant and fundamental group $\pi$ has a metric of positive scalar curvature. (The same result holds for nonspin manifolds, with $\Omega^{\text {spin }}$ replaced by $\Omega$ and without the condition on $\alpha(M)$.)

Theorem 2.4. Let $\pi$ be an elementary abelian 2-group, and let $n \geq 5$. Suppose $M^{n}$ is a closed oriented manifold with fundamental group $\pi$ and non-spin universal cover, and let $f: M \rightarrow B \pi$ be the classifying map for its universal covering. If the class $[M \stackrel{f}{\rightarrow} B \pi] \in H_{n}(B \pi, \mathbb{Z})$ is atoral, then $M$ has a metric with positive scalar curvature. In particular, if $n>\operatorname{rank} \pi$, then every oriented $n$-manifold with fundamental group $\pi$ and with non-spin universal cover has a metric of positive scalar curvature.

Remark 2.5. The case when $p$ is odd and $M$ is non-spin was already treated in [5]. For $p=2$, the case when $M$ is spin is very different from the non-spin case since $\Omega_{*}^{\text {spin }}\left(B(\mathbb{Z} / 2)^{r}\right)$ has a rather different structure from $\Omega_{*}^{S O}\left(B(\mathbb{Z} / 2)^{r}\right)$, and also because in the spin case there are many Dirac obstructions to positive scalar curvature.

\section{TOPOLOGICAL PRELIMINARIES}

Our basic tool will be the following Bordism/Homology Theorem, due to a combination of the work of Gromov-Lawson, Rosenberg-Stolz, Stolz, and Jung, which can be found in [14] and [15].

Theorem 3.1 (Bordism/Homology Theorem). Let $M^{n}$ be a (connected, closed) oriented manifold with $n=\operatorname{dim} M \geq 5$, and let $f: M \rightarrow B \pi$ be the classifying map for its universal covering. (In other words, $B \pi$ is a $K(\pi, 1)$-space, and $f$ induces an isomorphism on $\pi_{1}$. Existence and essential uniqueness of $f$ is guaranteed by obstruction theory.) 
(1) If $M$ is spin, $M$ admits a metric of positive scalar curvature if and only if there is some spin manifold of positive scalar curvature in the same spin bordism class as $M \stackrel{f}{\rightarrow} B \pi$, in fact if there is some spin manifold of positive scalar curvature representing the same class in $k_{n}(B \pi)$ as $M \stackrel{f}{\rightarrow} B \pi$.

(2) If the universal cover $\widetilde{M}$ of $M$ is non-spin (i.e., $w_{2}(\widetilde{M}) \neq 0$ ), $M$ admits a metric of positive scalar curvature if and only if there is some oriented manifold of positive scalar curvature in the same oriented bordism class as $M \stackrel{f}{\rightarrow} B \pi$, in fact if there is some oriented manifold of positive scalar curvature representing the same class in $H_{n}(B \pi, \mathbb{Z})$ as $M \stackrel{f}{\rightarrow} B \pi$.

Before proceeding further, we would like to remind the reader of some known results on $\Omega_{*}(B \pi)$ and $\Omega_{*}^{\text {spin }}(B \pi)$, where $\pi$ is an elementary abelian $p$-group of rank $r$ with $p$ an odd prime. Recall that

$$
\left(\Omega_{*}\right)_{(p)} \cong \mathbb{Z}_{(p)}\left[x_{4}, x_{8}, \ldots, x_{4 k}, \ldots\right]
$$

with $\left|x_{4 k}\right|=4 k$. Let $P=\mathbb{Z}_{(p)}\left[x_{4 i} \mid 2 i \neq p^{\ell}-1\right]$, and $M(P)$ denote the corresponding generalized Moore spectrum (just a wedge of shifted sphere spectra, localized at $p$ ). Then there is a splitting of the spectrum

$$
M S O_{(p)}=B P \wedge M(P) \cong \bigvee_{i_{1}, j_{1}, i_{2}, j_{2}, \cdots \geq 0} \Sigma^{4 i_{1}^{j_{1}}+4 i_{2}^{j_{2}}+\cdots} B P
$$

where $2 i_{1}, 2 i_{2}, \cdots \neq p^{\ell}-1$.

Here $B P_{*}=\mathbb{Z}_{(p)}\left[v_{1}, \ldots, v_{k}, \ldots\right],\left|v_{j}\right|=2\left(p^{j}-1\right)$. In particular, we have a $B P_{*^{-}}$ module isomorphism $\left(\Omega_{*}\right)_{(p)} \cong B P_{*} \otimes P$. Our goal is to describe the image of the map $\Omega_{*}(B \pi) \rightarrow H_{*}(B \pi ; \mathbb{Z})$ or $\Omega_{*}^{\text {spin }}(B \pi) \rightarrow k o_{*}(B \pi)$. But since $\pi$ is an elementary abelian $p$-group with $p$ odd, it is enough to work in the $p$-local category; furthermore, the natural map $M S O \rightarrow M$ Spin is a $p$-local equivalence, so $\widetilde{\Omega}_{*}^{\text {sin }}(B \pi) \cong \widetilde{\Omega}_{*}(B \pi)$. Furthermore, $\left(k o_{*}\right)_{(p)} \cong \mathbb{Z}_{(p)}\left[\omega_{4}\right]$ for $p$ odd, and the generators $x_{4 i}$ and $v_{j}$ may be chosen [3, Proposition 2.1] so that the map of rings $\Omega_{*}^{\text {spin }} \rightarrow k o_{*}$ corresponds to killing all the $v_{j}, j>1$, and $x_{4 i}, i>4$, and sending either $v_{1}$ (when $p=3$ ) or $x_{4}$ (when $p>3$ ) to $\omega_{4}$. (When $p>3$, then $v_{1} \mapsto c \omega_{4}^{(p-1) / 2}$ for some $c \neq 0$.) The map $\widetilde{\Omega}_{*}(B \pi) \rightarrow \widetilde{H}_{*}(B \pi ; \mathbb{Z})$ always factors through $\widetilde{B} P_{*}(B \pi)$, and so does the map $\widetilde{\Omega}_{*}^{\text {spin }}(B \pi) \rightarrow \widetilde{k} o_{*}(B \pi)$ when $p=3$. (When $p>3$, it factors through a wedge of $p-2$ copies of $\widetilde{B} P_{*}(B \pi)$.) So it is enough to work with $B P_{*}(B \pi)$. We need the following results on the structure of $B P_{*}(B \mathbb{Z} / p)$; see, say, 7]. We have that $B P^{*}\left(\mathbb{C P}^{\infty}\right)=B P^{*}[[x]]$, where $x \in B P^{2}\left(\mathbb{C P}^{\infty}\right)$ is the $(B P$ version of the $)$ first Chern class. Here we identify $B P^{-*}=B P_{*}$. Start with the standard fibration

$$
B \mathbb{Z} / p \longrightarrow \mathbb{C P}^{\infty} \stackrel{p}{\longrightarrow} \mathbb{C P}^{\infty},
$$

where the map $p: \mathbb{C P}^{\infty}=K(\mathbb{Z}, 2) \rightarrow K(\mathbb{Z}, 2)=\mathbb{C P}^{\infty}$ induces multiplication by $p$ on $\pi_{2}\left(\mathbb{C P}^{\infty}\right)$. Then $p^{*}: B P^{*}\left(\mathbb{C P}^{\infty}\right) \rightarrow B P^{*}\left(\mathbb{C P}^{\infty}\right)$ is given as

$$
p^{*}(x)=[p](x)=\sum_{i \geq 0} a_{i} x^{2 i+1}, \quad a_{i} \in B P_{4 i} .
$$

(We have re-indexed from the way things are written in 7], since if $p$ is odd, then $B P_{*}$ is concentrated in degrees divisible by 4.) In particular,

$$
B P^{*}(B \mathbb{Z} / p) \cong B P^{*}[[x]] /[p](x) .
$$


Let $I_{n}=\left(p, v_{1}, \ldots, v_{n-1}\right)$. The coefficients $a_{i}$ satisfy

- $a_{i} \in I_{n}$ for $i<\left(p^{n}-1\right) / 2$;

- $a_{\left(p^{n}-1\right) / 2} \equiv v_{n} \bmod I_{n} ;$ in particular, $a_{0}=p$, and $a_{i}=0$ for $0<i<$ $(p-1) / 2$, and $a_{(p-1) / 2}=v_{1}$.

The $B P_{*}$-module $N_{*}=\widetilde{B P}_{*}(B \mathbb{Z} / p)$ is generated by elements

$$
z_{2 m-1} \in B P_{2 m-1}(B \mathbb{Z} / p)
$$

(represented by the standard lens spaces), subject to the relations:

$$
R_{m}=\sum_{i=0}^{m-1} a_{i} z_{m-4 i}=0 .
$$

In particular, $N_{*}$ has homological dimension 1 as a $B P_{*}$-module. It was shown in [10] that $\widetilde{B P}_{*}\left(B(\mathbb{Z} / p)^{r}\right)$ may be computed via iterated applications of a Künneth formula involving $\cdot \otimes_{B P_{*}} N_{*}$ and $\Sigma \operatorname{Tor}_{B P_{*}}\left(\cdot, N_{*}\right)$. Let $L_{k}$ be the free $B P_{*}$-module on generators of degree $2 k, 1 \leq k \leq p^{k}-1$, and let $\pi=(\mathbb{Z} / p)^{r}$. It is shown in [7] that $\Sigma \operatorname{Tor}_{B P_{*}}\left(N_{*}, N_{*}^{\otimes^{j}}\right) \cong L_{j} \otimes N_{*}^{\otimes^{j}}$, and thus that there is a natural filtration on $\widetilde{B P}_{*}(B \pi)$, coming from the Landweber Künneth Theorem, for which the associated graded $B P_{*}$-module is a direct sum of tensor powers $N_{*}^{\otimes^{j}}, 1 \leq j \leq r$ (these correspond to pure product terms in the Künneth formula) and tensor products of tensor powers of $N$ with tensor powers of $L_{1}, \cdots, L_{r-1}$, where these terms have geometrical generators involving Toda brackets.

For future use, we also record a few more facts about the natural transformations $B P_{*} \rightarrow H_{*}$ or $\bigvee_{j=1}^{p-2} \Sigma^{4 j} B P_{*} \rightarrow k o_{*}$. Via these maps, the Eilenberg-Mac Lane spectrum $H=H \mathbb{Z}_{(p)}$ becomes a $B P$-module spectrum, so for ( $p$-local) spaces $X$ with $B P_{*}(X)$ of finite homological dimension over $B P_{*}$ there is a convergent universal coefficient spectral sequence [1, $\S 1,(\mathrm{UCT} 1)$ ]

$$
\operatorname{Tor}_{B P_{*}}^{s}\left(B P_{t}(X), \mathbb{Z}\right) \Rightarrow H_{s+t}(X, \mathbb{Z})
$$

for which one of the edge homomorphisms is

$$
E_{0, n}^{2}=B P_{n}(X) \otimes_{B P_{*}} \mathbb{Z} \rightarrow H_{n}(X, \mathbb{Z}) .
$$

The map $B P_{n}(X) \rightarrow H_{n}(X, \mathbb{Z})$ factors through this map (which simply kills $v_{1}$, $\left.v_{2}, \cdots\right)$. Similarly (again by [1, §1]) there is a universal coefficient spectral sequence

$$
\operatorname{Tor}_{B P_{*}\left[x_{4}\right]}^{*}\left(B P_{*}(X)\left[x_{4}\right], k o_{*}\right) \Rightarrow k o_{*}(X)
$$

for which one of the edge homomorphisms is

$$
B P_{*}(X)\left[x_{4}\right] \otimes_{B P_{*}} \mathbb{Z}\left[\omega_{4}\right] \rightarrow k o_{*}(X) .
$$

Notation 3.2. As in [3, we write $\mathfrak{k}_{*}(X)$ for $B P_{*}(X)\left[x_{4}\right] \otimes_{B P_{*}} \mathbb{Z}\left[\omega_{4}\right]$, the result of killing $v_{2}, v_{3}, \cdots$ and mapping $x_{4} \mapsto \omega_{4}, v_{1} \mapsto c \omega_{4}^{(p-1) / 2}$ for suitable $c \neq 0$. The Thom maps $B P_{n}(X) \rightarrow H_{n}(X, \mathbb{Z})$ and $B P_{n}(X) \rightarrow k o_{n}(X)$ thus factor through $\mathfrak{k}_{n}(X)$, though in general the map $\mathfrak{k}_{*}(X) \rightarrow k o_{*}(X)$ is neither injective nor surjective. However, one can check (from the $B P_{*}$-resolution of $N_{*}$ with relations (3.2)) that for $X=B \mathbb{Z} / p$, (3.4) collapses and gives an isomorphism $\mathfrak{k o}_{*}(B \mathbb{Z} / p) \cong$ $k o_{*}(B \mathbb{Z} / p)$. In particular, the map $\Omega_{*}^{\text {spin }}(B \mathbb{Z} / p) \rightarrow k o_{*}(B \mathbb{Z} / p)$ is surjective. 


\section{Proofs of the MAIN Results FOR $p$ ODD}

The following fact will be our key technical tool in the proof of Theorem 2.3 The homotopy functor $\mathfrak{k} \mathfrak{o}(\cdot)$ (not a homology theory) is defined in Notation [3.2.

Proposition 4.1. Let $\pi$ be an elementary abelian p-group of rank 2 , where $p$ is an odd prime. Then $\mathfrak{k}_{\text {odd }}(B \pi)$ is generated (as an abelian group) by the images of $\widetilde{\mathfrak{k}}_{*}(B \sigma)$, as $\sigma$ runs over the cyclic subgroups of $\pi$.

Proof. This is proved in 3], using explicit calculations of the eta-invariants of lens spaces.

Now we are ready to prove the following result.

Theorem 4.2. Let $\pi$ be an elementary abelian p-group of rank $r$, where $p$ is an odd prime. Then the image of $\Omega_{*}^{\mathrm{spin}}(B \pi)$ in $k o_{*}(B \pi)$ is generated (as an abelian group) by images of elements $x_{1} \otimes \cdots \otimes x_{j} \in \Omega_{*}^{\text {spin }}\left(B \sigma_{1}\right) \otimes \cdots \otimes \Omega_{*}^{\text {spin }}\left(B \sigma_{j}\right)$, with $\sigma_{1} \times \cdots \times \sigma_{j} \hookrightarrow \pi, j \leq r$, and with each $\sigma_{i}$ a cyclic p-group. (However, the embedding $\sigma_{1} \times \cdots \times \sigma_{j} \hookrightarrow \pi$ is not necessarily the "standard" one.)

Proof. We prove this by induction on the rank $r$. When $r=1$, the statement is trivially true, and when $r=2$, this is Proposition 4.1. Now assume the result for smaller values of $r$, and write $\pi=\pi^{\prime} \times \mathbb{Z} / p$, where $\pi^{\prime}$ has rank $r-1$. We use the $B P$-version of the diagram (4.1) in [5, Proof of Theorem 4.1]:

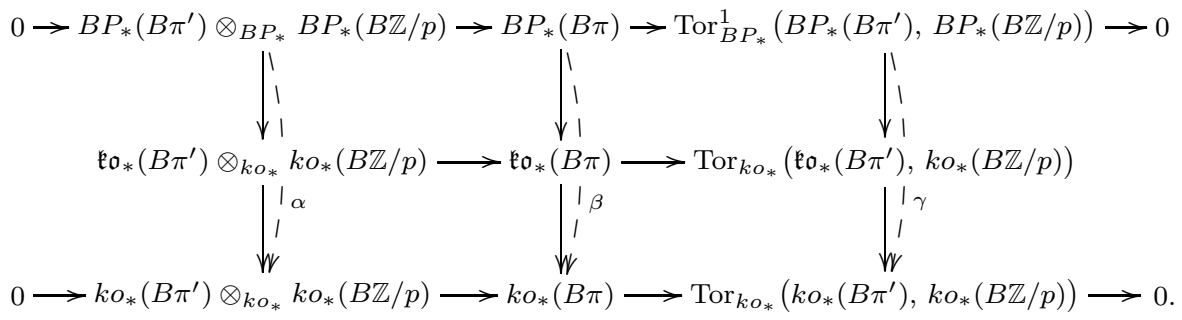

Here the bottom row comes from the Künneth Theorem in $k o_{*}$, which applies to this case (see again 1]) since $B \pi^{\prime}, B \mathbb{Z} / p$, and $B \pi$ are $p$-local for $p$ odd. In the top row, if $p>3$ one should really replace $B P_{*}$ by $B P\left[\omega_{4}\right]_{*}$ (but we've left off the $\left[\omega_{4}\right]$ to save space). The image of $\alpha$ is taken care of by the inductive hypothesis. Also by the inductive hypothesis, the image of $\gamma$ is generated by the images of

$$
\operatorname{Tor}_{B P[\omega]_{*}}^{1}\left(B P[\omega]_{*}\left(B \sigma_{1}\right) \otimes_{B P[\omega]_{*}} \cdots \otimes_{B P[\omega]_{*}} B P[\omega]_{*}\left(B \sigma_{j}\right), B P[\omega]_{*}(B \mathbb{Z} / p)\right)
$$

for the various subgroups $\sigma_{1} \times \cdots \times \sigma_{j} \hookrightarrow \pi^{\prime}$. The image of 4.2 is contained in a copy of the image of $B P[\omega]_{*}\left(B(\mathbb{Z} / p)^{r-1}\right)$ if $j<r-1$, which is also covered by the inductive hypothesis. So we may assume $j=r-1$. Thus we are reduced to studying the image of the map

$$
\gamma: \operatorname{Tor}_{B P_{*}}^{1}(\overbrace{N_{*} \otimes_{B P_{*}} \cdots \otimes_{B P_{*}} N_{*}}^{r-1}, N_{*})[\omega] \rightarrow \operatorname{Tor}_{k o_{*}}(\overbrace{P_{*} \otimes_{k o_{*}} \cdots \otimes_{k o_{*}} P_{*}}^{r-1}, P_{*}),
$$

where $P_{*}=\widetilde{k o_{*}}(B \mathbb{Z} / p)$ as a module over $k o_{*}=\mathbb{Z}[\omega]$. (Recall that we have localized at $p$. Also recall from Section $\left[3\right.$ that $P_{*}=N_{*}[\omega] \otimes_{B P[\omega]_{*}} k o_{*}$.) The $B P_{*}$-module on the left in (4.3) is computed in [7], Theorem 4.1]; this subquotient of $B P_{*}(B \pi)$ 
is a direct sum

$$
\operatorname{Tor}_{B P_{*}}^{1}\left(\left(N_{*}\right)^{\otimes^{r-1}}, N_{*}\right) \cong \bigoplus_{j=1}^{p^{r-1}-1}\left(N_{*}\right)^{\otimes^{r-1}} y_{2 j}
$$

of copies of $\left(N_{*}\right)^{\otimes^{r-1}}$, shifted up in degrees by $2 j, 0<j<p^{r-1}$. We need to show that the image of this graded group under (4.3) is generated by products of lens spaces.

Let $I=\left(i_{1}, \ldots, i_{r-1}\right)$ be a multi-index with all the $i_{k}$ 's odd, and let $z_{I}=$ $z_{i_{1}} \otimes \cdots \otimes z_{i_{r-1}}$ be the corresponding product of lens spaces (or the element of $\left(N_{*}\right)^{\otimes^{r-1}}$ or $\left(P_{*}\right)^{\otimes^{r-1}}$ represented by this product). Then we need to show that the image in $k o_{*}$ of $z_{I} y_{2 j} \in B P_{*}(B \pi)$ is represented by linear combinations of products of lens spaces, for $0<j<p^{r-1}$. Since the $B P_{*}$-module $N_{*}$ is given in terms of the generators $z_{m}$ and the relations $R_{m}$, the element $z_{I} y_{2 j}$ is represented by a linear combination of "matrix Toda brackets" [2, §5.10]

$$
\left\langle z_{i_{1}} \otimes \cdots \otimes z_{i_{k-1}} \otimes\left(z_{i_{k}}, z_{i_{k}-4}, \cdots\right) \otimes z_{i_{k+1}} \otimes \cdots \otimes z_{i_{r-1}}, A,\left(\begin{array}{c}
\vdots \\
z_{2 j-5} \\
z_{2 j-1}
\end{array}\right)\right\rangle
$$

possibly with smaller values of $i_{k}$, where $A$ is the matrix

$$
\left(\begin{array}{ccccc}
p & 0 & 0 & 0 & \ldots \\
a_{1} & p & 0 & 0 & \ldots \\
a_{2} & a_{1} & p & 0 & \ldots \\
a_{3} & a_{2} & a_{1} & p & \ldots \\
\vdots & \vdots & \vdots & \vdots & \ddots
\end{array}\right)
$$

encoding the relations (3.2). By the product property of Toda brackets [2, 2.1, axiom 3], 4.4 can be rewritten as

$$
\pm z_{i_{1}} \otimes \cdots \otimes z_{i_{k-1}} \otimes\langle\cdots\rangle \otimes z_{i_{k+1}} \otimes \cdots \otimes z_{i_{r-1}},
$$

where the bracket in the middle corresponds to something of rank 2 to which we can apply Proposition 4.1 This gives the inductive step.

Proof of Theorem 2.3. Now we can give the proof of the first main theorem. Recall that the atoral part of $\Omega_{*}^{\text {spin }}(B \pi)$ was defined in Definition 2.1 . By Theorem 4.2 it is enough to consider product classes

$$
\left(M_{1}^{i_{1}} \rightarrow B \mathbb{Z} / p\right) \times \cdots \times\left(M_{k}^{i_{k}} \rightarrow B \mathbb{Z} / p\right)
$$

in $\Omega_{i_{1}+\cdots+i_{k}}^{\text {spin }}\left(B(\mathbb{Z} / p)^{k}\right)$, as the image of $\Omega_{*}^{\text {spin }}(B \pi)$ in $k o_{*}(B \pi)$ is generated by the images of such classes under the maps $\Omega_{*}^{\text {spin }}\left(B(\mathbb{Z} / p)^{k}\right) \rightarrow \Omega_{*}^{\text {spin }}(B \pi)$ induced by embeddings $(\mathbb{Z} / p)^{k} \hookrightarrow \pi, k \leq \operatorname{rank} \pi=r$. But by Remark [2.2(a), the class (4.5) is toral exactly when $i_{1}=\cdots=i_{k}=1$, and atoral exactly when some $i_{j}>1$, and by Remark 2.2 (b), toral or atoral classes in $\Omega_{*}^{\text {spin }}\left(B(\mathbb{Z} / p)^{k}\right)$ map to toral or atoral classes in $\Omega_{*}^{\text {spin }}(B \pi)$. So the result follows from the fact that all atoral classes in $\widetilde{\Omega}_{*}^{\text {spin }}(B \mathbb{Z} / p)$ are represented by manifolds of positive scalar curvature. 


\section{Proofs of the main Results for $p=2$}

The parallel to Theorem 4.2 when $p=2$ is the following:

Theorem 5.1. Let $\pi$ be an elementary abelian 2-group. Then for any $n>1$, $H_{n}^{\text {atoral }}(B \pi, \mathbb{Z})$ is generated (as an abelian group) by classes of maps $f: M^{n} \rightarrow B \pi$, where $M$ is an oriented $n$-manifold of positive scalar curvature, and where $M$ admits an orientation-reversing isometry $c: M \rightarrow M$, admitting a fixed point, commuting up to homotopy with $f$.

Proof. Since $\pi$ is an elementary abelian 2-group and $M S O$ localized at 2 is an Eilenberg-Mac Lane spectrum (see [1] and [18, or [13, pp. 54-57] for a review of the literature) the map $\Omega_{*}(B \pi) \rightarrow H_{*}(B \pi, \mathbb{Z})$ is split surjective. Also, we know that $\widetilde{H}_{*}(B \pi, \mathbb{Z})$ embeds in $H_{*}\left(B \pi, \mathbb{F}_{2}\right)$, which pairs non-degenerately with

$$
H^{*}\left(B \pi, \mathbb{F}_{2}\right)=\mathbb{F}_{2}\left[x_{1}, \cdots, x_{r}\right],
$$

where $x_{1}, \cdots, x_{r}$ are cohomology generators each of degree 1 . So we will detect homology classes by means of pairing with this polynomial ring. By 5 , Proposition $5.2]$, for $n \geq 1$,

$$
\operatorname{dim}_{\mathbb{F}_{2}} H_{n}(B \pi, \mathbb{Z})=\sum_{j=1}^{n}(-1)^{n-j}\left(\begin{array}{c}
j+r-1 \\
r-1
\end{array}\right),
$$

while the toral subgroup has dimension $\left(\begin{array}{l}r \\ n\end{array}\right)$ if $n \leq r, 0$ if $n>r$. We will prove the result by induction on $r=\operatorname{rank} \pi$, with the condition regarding an orientationreversing isometry needed for the inductive step 1

To start the induction, take $r=1, \pi=\mathbb{Z} / 2$, and $B \pi=\mathbb{R} \mathbb{P}^{\infty}$. Note that $H_{n}(B \mathbb{Z} / 2, \mathbb{Z})$ vanishes for $n>0$ even and is generated for $n \geq 1$ by the class of $\mathbb{R P}^{n} \hookrightarrow \mathbb{R} \mathbb{P}^{\infty}$. This class is atoral when $n \geq 3$, exactly when it admits a metric of positive scalar curvature. Furthermore, $\mathbb{R P}^{n}$ ( $n$ odd) with its standard metric admits many orientation-reversing isometries, even of period 2 (for instance,

$$
\left[t_{0}, t_{1}, t_{2}, \cdots, t_{n}\right] \mapsto\left[t_{1}, t_{0}, t_{2}, \cdots, t_{n}\right]
$$

in homogeneous coordinates), all of which preserve the unique non-trivial element of $H^{1}\left(\mathbb{R} \mathbb{P}^{n}, \mathbb{Z} / 2\right)$ and thus commute up to homotopy with the map to $\mathbb{R} \mathbb{P}^{\infty}$. Any such isometry has a fixed point (by the Lefschetz Fixed Point Theorem). So this starts the induction.

Now assume the theorem is true for smaller values of $r$, and write $\pi=\pi^{\prime} \times \mathbb{Z} / 2$, where $\operatorname{rank} \pi^{\prime}=r-1$. By the Künneth Theorem, we have the short exact sequence

$$
\begin{aligned}
0 \rightarrow H_{n}\left(B \pi^{\prime}, \mathbb{Z}\right) \oplus \bigoplus_{j=1}^{[(n+1) / 2]} & H_{n-2 j+1}\left(B \pi^{\prime}, \mathbb{Z}\right) \otimes_{\mathbb{Z}} H_{2 j-1}(B \mathbb{Z} / 2, \mathbb{Z}) \rightarrow H_{n}(B \pi, \mathbb{Z}) \\
& \rightarrow \bigoplus_{j=1}^{[n / 2]} \operatorname{Tor}_{\mathbb{Z}}\left(H_{n-2 j}\left(B \pi^{\prime}, \mathbb{Z}\right), H_{2 j-1}(B \mathbb{Z} / 2, \mathbb{Z})\right) \rightarrow 0,
\end{aligned}
$$

\footnotetext{
${ }^{1}$ Actually it would be enough for our purposes to have an orientation-reversing diffeomorphism $c$ such that the metric and its pull-back under $g$ lie in the same connected component of the space of metrics of positive scalar curvature. But this condition, while weaker, is more burdensome to check.
} 
which splits (unnaturally). The "tensor" terms on the left in (5.1) are clearly no problem, as they are represented by classes of maps $M^{n} \rightarrow B \pi^{\prime} \times \mathrm{pt} \hookrightarrow B \pi^{\prime} \times$ $B \mathbb{Z} / 2$ or $M^{n-2 j+1} \times \mathbb{R P}^{2 j-1} \stackrel{f \times \iota}{\longrightarrow} B \pi^{\prime} \times B \mathbb{Z} / 2$, where $\iota: \mathbb{R} \mathbb{P}^{2 j-1} \hookrightarrow \mathbb{R} \mathbb{P}^{\infty}$. By the inductive hypothesis, $H_{*}^{\text {atoral }}\left(B \pi^{\prime}, \mathbb{Z}\right)$ is represented by manifolds of positive scalar curvature, hence so is $H_{*}^{\text {atoral }}\left(B \pi^{\prime}, \mathbb{Z}\right) \otimes H_{*}(B \mathbb{Z} / 2, \mathbb{Z})$. Similarly anything in $H_{*}\left(B \pi^{\prime}, \mathbb{Z}\right) \otimes H_{2 j-1}(B \mathbb{Z} / 2, \mathbb{Z})$ admits a metric of positive scalar curvature if $j>1$. On the other hand, manifolds of the form $M^{n}$ or $M^{n-1} \times S^{1}$, with $M$ toral, are themselves toral. Furthermore, the required orientation-reversing isometry on $M^{n-2 j+1} \times \mathbb{R} \mathbb{P}^{2 j-1}$ can be constructed from one on either $M$ or $\mathbb{R} \mathbb{P}^{2 j-1}$.

So that leaves only the Tor terms $H_{n-2 j}\left(B \pi^{\prime}, \mathbb{Z}\right) * H_{2 j-1}(B \mathbb{Z} / 2, \mathbb{Z})$ on the right in (5.1) to worry about. These terms are represented (non-canonically) by homology Toda brackets $\left\langle\left[M^{n-2 j}\right], 2, z_{2 j-1}\right\rangle$. We will distinguish two cases: (a) the case where either $j>1$ or the class $\left[M^{n-2 j}\right] \in H_{n-2 j}\left(B \pi^{\prime}\right)$ is atoral, and (b), the exceptional case of $H_{n-2}^{\text {toral }}\left(B \pi^{\prime}, \mathbb{Z}\right) * H_{1}(B \mathbb{Z} / 2, \mathbb{Z})$. First consider the case (a). We shall show that representatives for these terms can be constructed as twisted products, i.e., fiber bundles, of the form

$$
M^{n-2 j} \times \mathbb{R P}^{2 j-1} \rightarrow N^{n} \rightarrow S^{1},
$$

where $g: M \rightarrow B \pi^{\prime}$ represents a class in $H_{n-2 j}\left(B \pi^{\prime}, \mathbb{Z}\right)$. By assumption, $M$ admits an orientation-reversing isometry $c_{1}$ with a fixed point, and similarly $\mathbb{R} \mathbb{P}^{2 j-1}$ admits an orientation-reversing isometry $c_{2}$ with a fixed point. Let $c=c_{1} \times c_{2}$; this is an isometry of $M^{n-2 j} \times \mathbb{R} \mathbb{P}^{2 j-1}$ that preserves orientation. Thus if $N$ denotes the mapping torus of $c, N$ is an oriented manifold fitting into a fiber sequence (5.2). Also note that $N$ contains within it two submanifolds $N_{1}^{n-2 j+1}$ and $N_{2}^{2 j}$, the mapping tori of $c_{1}$ and $c_{2}$, respectively. Since $c_{1}$ and $c_{2}$ reverse orientation, $N_{1}$ and $N_{2}$ are not orientable. Now consider the cohomology ring $H^{*}\left(N, \mathbb{F}_{2}\right)$. This ring has a filtration (associated to the Leray-Serre spectral sequence

$$
H^{*}\left(S^{1}, H^{*}\left(M \times \mathbb{R P}^{2 j-1}, \mathbb{F}_{2}\right)\right) \Rightarrow H^{*}\left(N, \mathbb{F}_{2}\right),
$$

which collapses since $S^{1}$ is 1-dimensional) for which the associated graded ring is just the tensor product

$$
H^{*}\left(M \times \mathbb{R P}^{2 j-1}, \mathbb{F}_{2}\right) \otimes H^{*}\left(S^{1}, \mathbb{F}_{2}\right) \cong H^{*}\left(M, \mathbb{F}_{2}\right)[v, w] /\left(v^{2 j}, w^{2}\right) .
$$

However, it will be important to note that the actual product structure on $H^{*}\left(N, \mathbb{F}_{2}\right)$ is different. To prove this, consider for instance the inclusion $\iota: N_{2} \hookrightarrow N .\left(N_{2}\right.$ is the mapping torus of the restriction of $c$ to $\{\mathrm{pt}\} \times \mathbb{R P}^{2 j-1}$, with the base point in $M$ chosen to be a fixed point of $c_{1}$. The case of $N_{1} \hookrightarrow N$ is similar, except that our knowledge of the cohomology ring of $M$, and thus of the cohomology ring of $N_{1}$, is less explicit.) Note that $H^{*}\left(N_{2}, \mathbb{F}_{2}\right)$ is generated by two elements of degree 1 , say $v$ and $w$. The first of these restricts to a generator of $H^{*}\left(\mathbb{R P}^{2 j-1}, \mathbb{F}_{2}\right)$, and the second restricts to a generator of $H^{*}\left(S^{1}, \mathbb{F}_{2}\right)$ in the cohomology of the base. But since $N_{2}$ is not orientable, it has a non-trivial first $\mathbb{Z} / 2$-characteristic class $w_{1}=v_{1}$. Since the first $\mathrm{Wu}$ class $v_{1}$ is characterized by the relation $\operatorname{Sq}^{1}(x)=x \cdot v_{1}$ for $x$ a cohomology class of codimension 1 , and since the codimension- 1 cohomology is generated by $v^{2 j-1}$ and $v^{2 j} w$, one of $\mathrm{Sq}^{1}\left(v^{2 j-1}\right)$ and $\mathrm{Sq}^{1}\left(v^{2 j} w\right)$ must be non-zero. But $\mathrm{Sq}^{1}$ is a derivation and $\mathrm{Sq}^{1}(v)=v^{2}, \mathrm{Sq}^{1}(w)=w^{2}=0$, so

$$
\mathrm{Sq}^{1}\left(v^{2 j} w\right)=(2 j) v^{2 j-1} \mathrm{Sq}^{1}(v) w+v^{2 j} \mathrm{Sq}^{1}(w)=0+0=0 .
$$


Thus $\operatorname{Sq}^{1}\left(v^{2 j-1}\right)=(2 j-1) v^{2 j-2} \operatorname{Sq}^{1}(v)=v^{2 j} \neq 0$. Since $v^{2 j}$ maps to 0 in the associated graded ring of $H^{*}\left(N, \mathbb{F}_{2}\right)$, this forces $v^{2 j}=v^{2 j-1} w$ (which shows incidentally that $v_{1}=w$, which one can check by other means). Thus $v^{2 j}$ pairs non-trivially with the $\mathbb{Z} / 2$-fundamental class $\left[N_{2}\right]$. Now a map $f: N \rightarrow B \pi=B \pi^{\prime} \times B \mathbb{Z} / 2$ is determined by choosing values for $f^{*}$ applied to a basis for $H^{1}\left(B \pi, \mathbb{F}_{2}\right)=$ $H^{1}\left(B \pi^{\prime}, \mathbb{F}_{2}\right) \oplus H^{1}\left(B \mathbb{Z} / 2, \mathbb{F}_{2}\right)$. So we define $f$ by requiring that $f^{*}(x)=v$, where $0 \neq$ $x \in H^{1}\left(B \mathbb{Z} / 2, \mathbb{F}_{2}\right)$, and that $f^{*}: H^{1}\left(B \pi^{\prime}, \mathbb{F}_{2}\right) \rightarrow H^{1}\left(N, \mathbb{F}_{2}\right)$ lift $g^{*}: H^{1}\left(B \pi^{\prime}, \mathbb{F}_{2}\right) \rightarrow$ $H^{1}\left(M, \mathbb{F}_{2}\right)$. From the calculation we just made, we see that for $\delta \in H^{*}\left(B \pi^{\prime}, \mathbb{F}_{2}\right)$, we have

$$
\begin{aligned}
\left\langle\delta \cdot x^{k}, f_{*}([N])\right\rangle & =\left\langle f^{*}\left(\delta \cdot x^{k}\right),[N]\right\rangle \\
& =\left\langle f^{*}(\delta) \cdot v^{k},[N]\right\rangle \\
& = \begin{cases}1, & k=2 j \text { and }\left\langle\delta, g_{*}([N])\right\rangle \neq 0, \\
1, & k=2 j-1 \text { and }\left\langle f^{*}(\delta),\left[N_{1}\right]\right\rangle \neq 0, \\
0, & \text { otherwise. }\end{cases}
\end{aligned}
$$

The fact that $f_{*}([N])$ pairs non-trivially with $\delta \cdot x^{2 j}$, where $\left\langle\delta, g_{*}([N])\right\rangle \neq 0$, shows that the class of $N \stackrel{f}{\rightarrow} B \pi$ is not in the "tensor part" of the homology, and a dimension count shows that we get all the "Tor terms" by this construction. Now we just need to check that $N$ has all the right properties. Since $c$ preserves a metric of positive scalar curvature on $M^{n-2 j} \times \mathbb{R P}^{2 j-1}$, the product metric (of positive scalar curvature) on $M^{n-2 j} \times \mathbb{R}^{2 j-1} \times[0,1]$ descends to a metric of positive scalar curvature on $N$ (under the identification of $M^{n-2 j} \times \mathbb{R P}^{2 j-1} \times\{0\}$ with $\left.M^{n-2 j} \times \mathbb{R P}^{2 j-1} \times\{1\}\right)$. The required orientation-reversing isometry is induced by $c_{1}$ or $c_{2}$.

Now consider the exceptional case (b), i.e., the case of

$$
H_{n-2}^{\text {toral }}\left(B \pi^{\prime}, \mathbb{Z}\right) * H_{1}(B \mathbb{Z} / 2, \mathbb{Z}) .
$$

In this case, the construction above does not yield a manifold of positive scalar curvature, so we need to do something different. Since $H_{n-2}^{\text {toral }}\left(B \pi^{\prime}, \mathbb{Z}\right)$ is the image of the class of $T^{n-2} \rightarrow B(\mathbb{Z} / 2)^{n-2}$ under a map $(\mathbb{Z} / 2)^{n-2} \rightarrow \pi^{\prime}$ (cf. Remark 2.2(b)), it suffices to suppose $r=n-1$ and consider the homology Toda bracket

$$
\left\langle z_{1}^{n-2}, 2, z_{1}\right\rangle=z_{1}^{n-3}\left\langle z_{1}, 2, z_{1}\right\rangle .
$$

(We have again used 2, 2.1, axiom 3].) If $\left\langle z_{1}, 2, z_{1}\right\rangle \in H_{3}\left(B(\mathbb{Z} / 2)^{2}, \mathbb{F}_{2}\right)$ can be represented by a manifold $P^{3} \rightarrow \mathbb{R} \mathbb{P}^{\infty} \times \mathbb{R} \mathbb{P}^{\infty}$ of positive scalar curvature, admitting an orientation-reversing isometry commuting up to homotopy with the map to $\mathbb{R} \mathbb{P}^{\infty} \times \mathbb{R} \mathbb{P}^{\infty}$, then $T^{n-3} \times P^{3}$, with the obvious map to $B \pi$, will satisfy the required conditions. But $H_{3}(B \pi, \mathbb{Z})$ turns out to be spanned by $z_{3} \otimes 1$ and $1 \otimes z_{3}$ together with the class of the diagonal embedding $\Delta$ of $\mathbb{R} \mathbb{P}^{3}$ in $\mathbb{R} \mathbb{P}^{\infty}$, as one can see by observing that

$$
\Delta^{*}: H^{*}(B \pi, \mathbb{Z} / 2)=\mathbb{F}_{2}[u, v] \rightarrow H^{*}\left(\mathbb{R P}^{3}, \mathbb{Z} / 2\right)=\mathbb{F}_{2}[x] /\left(x^{4}\right)
$$

sends both of the generators $u$ and $v$ to $x$, so that $\Delta_{*}\left(\left[\mathbb{R}^{3}\right]\right)$ pairs non-trivially with both $u^{2} v$ and $v^{2} u$, and thus cannot be in the span of the classes $z_{3} \otimes 1$ and $1 \otimes z_{3}$. Thus $\Delta_{*}\left(z_{3}\right)$ can be chosen as a representative for a generator of $\operatorname{Tor}_{\mathbb{Z}}\left(H_{1}\left(B \mathbb{Z} / 2, \mathbb{F}_{2}\right), H_{1}\left(B \mathbb{Z} / 2, \mathbb{F}_{2}\right)\right)$, and it clearly satisfies our requirements. This completes the inductive step. 
Proof of Theorem 2.4 Theorem 2.4 now follows immediately from combining Theorem 5.1 and Theorem 3.1 (2).

Remark 5.2 (added after this paper was accepted). Michael Joachim has now shown [6] that if $\pi$ is an elementary abelian 2-group, every toral class in $H_{n}(B \pi, \mathbb{Z}), n \geq 3$, is represented by an oriented manifold of positive scalar curvature. Combining this result with Theorem 2.4 yields the following stronger result:

Theorem 5.3. Let $\pi$ be an elementary abelian 2-group, and let $n \geq 5$. Then every oriented $n$-manifold with fundamental group $\pi$ and with non-spin universal cover has a metric of positive scalar curvature.

In other words, the Gromov-Lawson-Rosenberg Conjecture holds for oriented manifolds with non-spin universal cover and fundamental group an elementary abelian 2-group.

\section{REFERENCES}

[1] J. F. Adams, Lectures on generalised cohomology, in Category Theory, Homology Theory and their Applications, III (Battelle Institute Conference, Seattle, Wash., 1968, Vol. 3), Lecture Notes in Math., vol. 99, Springer, Berlin, 1969, pp. 1-138; reprinted in The Selected Works of J. Frank Adams, vol. 1, J. P. May and C. B. Thomas, eds., Cambridge Univ. Press, Cambridge, 1992, pp. 377-514. MR0251716 (40:4943)

[2] J. C. Alexander, Cobordism Massey products, Trans. Amer. Math. Soc. 166 (1972), 197-214. MR 0293623(45:2700)

[3] B. Botvinnik and P. Gilkey, The eta invariant and the Gromov-Lawson conjecture for elementary abelian groups of odd order, Topology Appl. 80 (1997), no. 1-2, 43-53. MR 1469465 (99f:58194)

[4] B. Botvinnik, P. Gilkey, and S. Stolz, The Gromov-Lawson-Rosenberg conjecture for groups with periodic cohomology, J. Differential Geom. 46 (1997), no. 3, 374-405. MR1484887 (98i:58227)

[5] B. Botvinnik and J. Rosenberg, The Yamabe invariant for non-simply connected manifolds, J. Differential Geom. 62 (2002), no. 2, 175-208. MR1988502

[6] M. Joachim, Toral classes and the Gromov-Lawson-Rosenberg Conjecture for elementary abelian 2-groups, preprint no. 262, SFB 478 Geometrische Strukturen in der Mathematik, Univ. of Münster, 2003. Available at http://wwwath. uni-muenster.de/math/inst/sfb/about/publ/.

[7] D. C. Johnson and S. W. Wilson, The Brown-Peterson homology of elementary p-groups, Amer. J. Math. 107 (1985), no. 2, 427-453. MR0784291|(86j:55008)

[8] D. Joyce, Compact Manifolds with Special Holonomy, Oxford Univ. Press, Oxford, 2000. MR 1787733 (2001k:53093)

[9] S. Kwasik and R. Schultz, Positive scalar curvature and periodic fundamental groups, Comment. Math. Helv. 65 (1990), no. 2, 271-286. MR1057244 (91k:57027)

[10] P. Landweber, Künneth formulas for bordism theories, Trans. Amer. Math. Soc. 121 (1966), no. 1, 242-256. MR 0192503 (33:728)

[11] I. Madsen and R. J. Milgram, The classifying spaces for surgery and cobordism of manifolds, Ann. of Math. Studies, vol. 92, Princeton Univ. Press, Princeton, N.J., 1979. MR0548575 (81b:57014)

[12] J. Rosenberg, The $K O$-assembly map and positive scalar curvature, in Algebraic Topology Poznań 1989, Lecture Notes in Math., vol. 1474, Springer, Berlin, 1991, pp. 170-182. MR 1133900 (92m:53060)

[13] J. Rosenberg, Reflections on C. T. C. Wall's work on cobordism, in Surveys on Surgery Theory: Volume 2, S. Cappell, A. Ranicki, and J. Rosenberg, eds., Annals of Math. Studies, vol. 149, Princeton Univ. Press, Princeton, NJ, 2001, pp. 49-61. MF.1818771

[14] J. Rosenberg and S. Stolz, A "stable" version of the Gromov-Lawson conjecture, in The Čech Centennial: Proc. Conf. on Homotopy Theory, M. Cenkl and H. Miller, eds., Contemp. Math. 181 (1995), Amer. Math. Soc., Providence, RI, pp. 405-418. MR1321004 (96m:53042) 
[15] J. Rosenberg and S. Stolz, Metrics of positive scalar curvature and connections with surgery, in Surveys on Surgery Theory: Volume 2, S. Cappell, A. Ranicki, and J. Rosenberg, eds., Annals of Math. Studies, vol. 149, Princeton Univ. Press, Princeton, NJ, 2001, pp. 353-386. MR:1818778(2002f:53054)

[16] R. Schultz, Positive scalar curvature and odd order abelian fundamental groups, Proc. Amer. Math. Soc. 125 (1997), no. 3, 907-915. MR.1363184 (97j:53041)

[17] S. Stolz, Positive scalar curvature metrics - existence and classification questions, in Proceedings of the International Congress of Mathematicians, Vol. 1 (Zürich, 1994), Birkhäuser, Basel, 1995, pp. 625-636. MF.1403963 (98h:53063)

[18] L. R. Taylor, 2-local cobordism theories, J. London Math. Soc. (2) 14 (1976), no. 2, 303-308. MR0431142(55:4144)

Department of Mathematics, University of Oregon, Eugene, Oregon 97403-1222

E-mail address: botvinn@poincare.uoregon.edu

$U R L$ : http://darkwing.uoregon.edu/ ${ }^{\text {botvinn }}$

Department of Mathematics, University of Maryland, College Park, Maryland 20742-4015

E-mail address: jmr@math.umd.edu

$U R L:$ http://www.math.umd.edu/ ${ }^{j m r}$ 